\title{
Validation of the Slow Off-Kinetics of Sirtuin-Rearranging Ligands (SirReals) by Means of Label-Free Electrically Switchable Nanolever Technology**
}

\author{
Matthias Schiedel ${ }^{+},{ }^{[a, b]}$ Herwin Daub $^{+},{ }^{[c, d]}$ Aymelt Itzen, $^{[d, e]}$ and Manfred Jung ${ }^{*[b]}$
}

\begin{abstract}
We have discovered the sirtuin-rearranging ligands (SirReals) to be highly potent and selective inhibitors of the $\mathrm{NAD}^{+}$-dependent lysine deacetylase Sirt2. Using a biotinylated SirReal in combination with biolayer interferometry, we previously observed a slow dissociation rate of the inhibitor-enzyme complex; this had been postulated to be the key to the high affinity and selectivity of SirReals. However, to attach biotin to the SirReal core, we introduced a triazole as a linking moiety; this
\end{abstract}

was shown by X-ray co-crystallography to interact with Arg97 of the cofactor binding loop. Herein, we aim to elucidate whether the observed long residence time of the SirReals is induced mainly by triazole incorporation or is an inherent characteristic of the SirReal inhibitor core. We used the novel labelfree switchSENSE ${ }^{\circledR}$ technology, which is based on electrically switchable DNA nanolevers, to prove that the long residence time of the SirReals is indeed caused by the core scaffold.

\section{Introduction}

Proteins are subjected to various post-translational modifications (PTMs). These modifications enable the fine-tuned regulation of protein activity, localization, interaction, and stability. ${ }^{[1]}$ With similar complexity and cellular importance to phosphorylation, acetylation of the $\varepsilon$-amine of lysine residues has emerged as one of the most abundant protein PTMs. ${ }^{[2]}$ Lysine is acetylated by lysine acetyltransferases (KATs); the group is re-

\section{[a] Dr. M. Schiedel}

Department of Chemistry and Pharmacy

Medicinal Chemistry, Friedrich Alexander University Erlangen-Nürnberg Nikolaus-Fiebiger-Strasse 10, 91058 Erlangen (Germany)

[b] Dr. M. Schiedel, ${ }^{+}$Prof. Dr. M. Jung

Institute of Pharmaceutical Sciences, Albert-Ludwigs-Universität Freiburg Albertstrasse 25, 79104 Freiburg im Breisgau (Germany)

E-mail: manfred.jung@pharmazie.uni-freiburg.de

[c] H. Daub

Dynamic Biosensors $\mathrm{GmbH}$

Lochhamer Strasse 15, 82152 Martinsried (Germany)

[d] H. Daub, ${ }^{+}$Prof. Dr. A. Itzen

Center for Integrated Protein Science Munich

Technische Universität München, Department of Chemistry Lichtenbergstrasse 4, 85748 Garching (Germany)

[e] Prof. Dr. A. Itzen

Department of Biochemistry and Signal Transduction University Medical Centre Hamburg-Eppendorf (UKE) Martinistrasse 52, 20246 Hamburg (Germany)

$\left.{ }^{[+}\right]$These authors contributed equally to this work.

[**] A previous version of this manuscript has been deposited on a preprint server (https://doi.org/10.26434/chemrxiv.9691730.v1).

$\square$ Supporting information and the ORCID identification numbers for the

(D) authors of this article can be found under https://doi.org/10.1002/ cbic.201900527.

of ( 2019 The Authors. Published by Wiley-VCH Verlag GmbH \& Co. KGaA This is an open access article under the terms of the Creative Commons Attribution Non-Commercial License, which permits use, distribution and reproduction in any medium, provided the original work is properly cited and is not used for commercial purposes. moved by lysine deacetylases (KDACs). ${ }^{[3]}$ In addition to acetyl, longer acyl chains such as propionyl, butyryl, and myristoyl, or acyl groups derived from dicarboxylic acids such as malonyl, succinyl, or glutaryl can be installed and removed by the aforementioned lysine-modifying enzymes. ${ }^{[4]}$ However, it is noteworthy that there is also a considerable amount of nonenzymatic lysine acylation. ${ }^{[5]}$

Eighteen different KDACs have been identified in the human genome, and grouped into four classes according to their sequence homology. ${ }^{[6]}$ Sirtuins, which constitute the class III KDACs, are very special members of the KDAC family. Whereas the class I, II, and IV deacetylases are $\mathrm{Zn}^{2+}$-dependent amidohydrolases, the seven human sirtuin isotypes (Sirt1 to -7) share an $\mathrm{NAD}^{+}$-dependent catalytic mechanism. In the course of the catalytic reaction, sirtuins undergo a rearrangement process from the so-called "open conformation" of the apo enzyme to the "closed conformation" of the (pseudo-)substrate-bound state. ${ }^{[7]}$ The isotype Sirt2 is predominantly localized in the cytoplasm and has been shown to deacetylate a variety of substrates, such as $\alpha$-tubulin, ${ }^{[8]}$ BubR $1,{ }^{[9]}$ p53, ${ }^{[10]}$ elF5A, ${ }^{[11]}$ and $N F \kappa B .{ }^{[12]}$ Sirt2-dependent deacetylation has a major impact on cell-cycle regulation, ${ }^{[8]}$ autophagy, ${ }^{[13]}$ peripheral myelination, ${ }^{[14]}$ and immune and inflammatory responses. ${ }^{[15]}$ In addition to deacetylation, Sirt2 catalyzes the removal of long-chain fatty acids, with an even higher catalytic efficiency $\left(k_{\mathrm{cat}} / K_{\mathrm{m}}\right)$ reported for demyristoylation than for deacetylation. ${ }^{[16]}$ However, a number of recent reports also imply that the overall cellular agenda of Sirt2 is not only dependent on its catalytic activity but also on its protein-protein interactions (PPI) with binding partners such as $\mathrm{KDAC6}^{[8]}$ or TTTP/p25. ${ }^{[17]}$ Dysregulation of Sirt2 has been associated with several disease states, including bacterial infections, ${ }^{[15 b]}$ type II diabetes, ${ }^{[18]}$ neurodegenerative diseases, ${ }^{[19]}$ and cancer ${ }^{[20]}$ thereby highlighting Sirt2 as a promising target for pharmaceutical intervention. However, for 
A)<smiles>[R]Cc1cnc(NC(=O)CSc2nc(C)cc(C)n2)s1</smiles>

Compound

$\mathrm{R}$

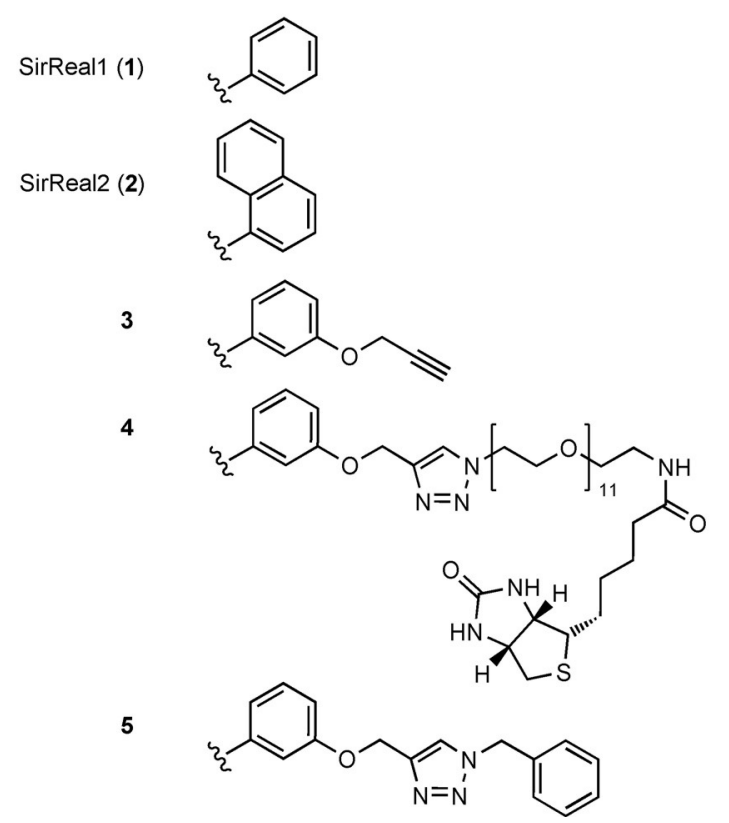

B)

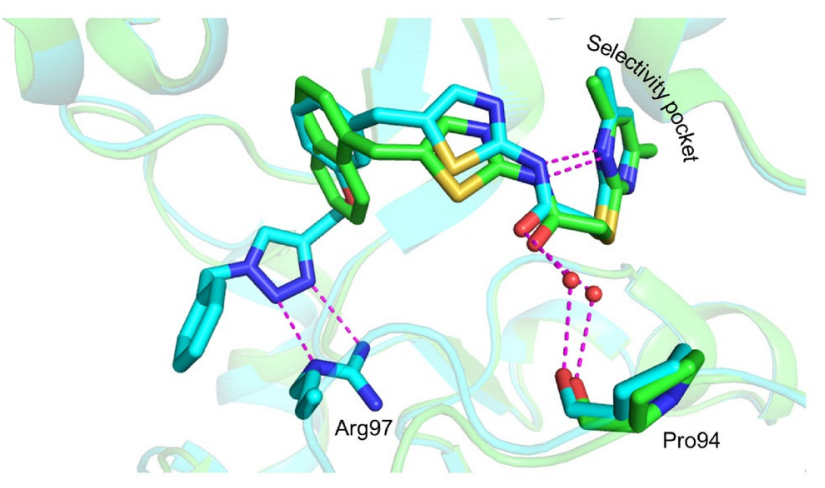

C)

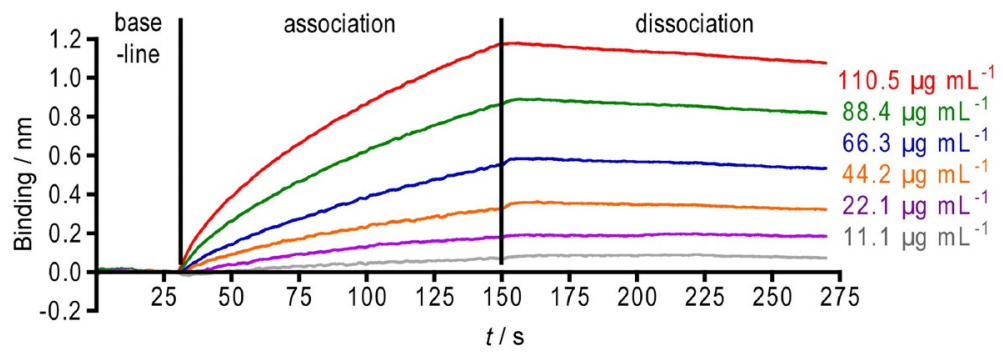

Figure 1. SirReals bind to Sirt2 by rearranging the active site resulting in slow off-kinetics. A) Chemical structures of selected SirReals $1-3$, the SirReal-derived affinity probe 4 , and the triazole-based SirReal analogue $5 .^{[24 a, 25]}$ B) Overlay of the binding modes of 2 (green, PDB ID: 4RMG) and 5 (cyan, PDB ID: 5DY5) binding to Sirt2. ${ }^{[24 a, 25]}$ Hydrogen bonds are shown as dashed purple lines. Interacting residues are shows as sticks and interacting water molecules as red spheres. In terms of clarity, residues 103-112 are omitted. An illustration of the binding modes of $\mathbf{2}$ and $\mathbf{5}$ covering a larger segment of the structures is provided in Figure S1. C) Representative biolayer interferometry sensorgram showing different concentrations of Sirt2 binding to the immobilized SirReal-derived affinity probe (5); adapted with permission from ref. [25]. Copyright: 2016, Wiley-VCH.

some disease scenarios, including Huntington's disease ${ }^{[21]}$ and some cancer types, ${ }^{[22]}$ it has not yet been finally clarified whether Sirt2 has to be up- or downregulated or even inhibited to ameliorate specific disease conditions. The urgent need for suitable tool compounds to further investigate the cellular effects of Sirt2 deacetylation and validate Sirt2 as a drug target led to the discovery of a number of drug-like Sirt2-selective small-molecule inhibitors, which have been reviewed elsewhere. ${ }^{[23]}$

Recently, we have discovered a new class of highly Sirt2-selective inhibitors. ${ }^{[24]}$ These compounds result in Sirt2 inhibition in the low-micromolar to nanomolar range, whereas no detectable inhibition $\left(\mathrm{IC}_{50}>100 \mu \mathrm{M}\right)$ can be observed for their close homologues Sirt1 and Sirt3 or the other isotypes. ${ }^{[2-25]}$ The cocrystal structures of Sirt2 in complex with 1 or 2 (Figure $1 \mathrm{~A}$ ) were the first crystal structures of Sirt2 complexed with Sirt2selective drug-like inhibitors. ${ }^{[24]}$ The data revealed a unique mode of inhibition that is characterized by a major rearrangement of the active site of Sirt2 upon ligand binding (Figure 1 B and Figure $\mathrm{S} 1$ in the Supporting Information). As a result of the rearrangement, a new binding pocket is formed by two loops of the hinge region, which connects the Rossmann fold domain with the zinc-binding domain. ${ }^{[24]}$ This phenomenon was only observed for Sirt2, and has been attributed to the unique flexibility of Sirt2 in this special region of the active site. Thus, inhibitors of this class were referred to as sirtuin-rearranging ligands (SirReals), and the induced-fit binding pocket was termed the selectivity pocket, as it was identified to be the key to Sirt2 selectivity. ${ }^{[24 a]}$ Very soon after we had reported the existence of the selectivity pocket in Sirt2, it was shown that this pocket accommodates the long-chain fatty acid of a myristoyl substrate. ${ }^{[26]}$ Meanwhile, other Sirt2 inhibitors were also shown to gain their isotype selectivity by binding to the selectivity pocket. ${ }^{[27]}$ Intrigued by the SirReal-Sirt2 co-crystal structures showing that ligand binding to the selectivity pocket prevents the switch from the open to the closed conformation, we hypothesized that the SirReals wedge Sirt2 into its open ("locked-open") conformation and are trapped by this process in return.

Therefore, we studied the binding kinetics of the SirRealSirt2 interaction. Initial attempts to determine the on- and offkinetics by means of isothermal titration calorimetry (ITC) failed; this might be linked to the fact that the binding enthalpy was overlaid by the energy contributions associated with the rearrangement process. Thus, we conjugated a propargylated SirReal analogue (3) with an azido-functionalized biotin to obtain the SirReal-derived affinity probe with a triazole linkage (4). This probe was submitted for binding kinetics studies 
with biolayer interferometry. Our kinetic measurements, which revealed very slow dissociation kinetics (Figure $1 \mathrm{C}$ ), suggested that Sirt2 indeed traps the bound SirReal in its active site as a consequence of its conformational adaption upon ligand binding. This results in a long residence time of the ligand on its target (i.e., the lifetime of the ligand-protein complex) and causes slow dissociation (Figure $1 \mathrm{C}$ ). ${ }^{[25,28]}$ The finding that conformational adaption of the targeted protein upon ligand binding can greatly influence residence time is consistent with the reported literature. ${ }^{[29]}$ Moreover, increasing the residence time has been proposed as a key strategy for optimizing cellular activity in drug design. ${ }^{[30]}$

However, as the co-crystal structure of Sirt2 in complex with the triazole-based SirReal $\mathbf{5}$ illustrates, the triazole moiety, which was introduced during the labeling process as a linker moiety, is also involved in Sirt2 binding through specific interactions. The triazole forms hydrogen bonds with Arg97 of the cofactor binding loop (Figure 1B). To interrogate whether

A)

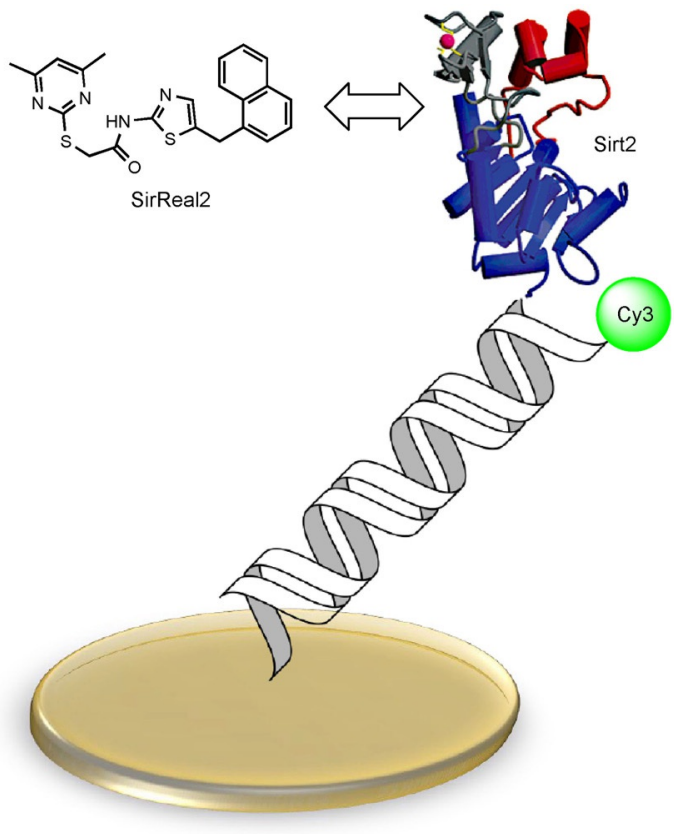

C)

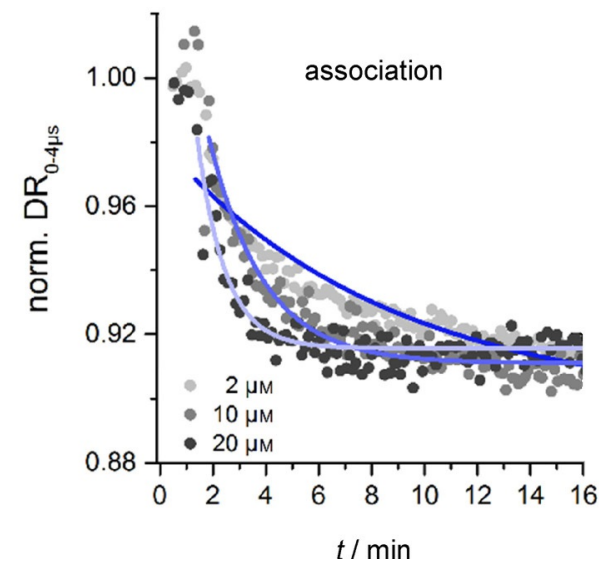

the long residence time of the Sirt2 affinity probe 4 is a result of the introduction of the triazole moiety or can be mainly attributed to the SirReal core, we sought a technology that enabled binding kinetics to be determined in a label-free approach.

\section{Results and Discussion}

In order to study the binding kinetics ( $k_{\text {on, }} k_{\text {off }}$ rate constants) of the Sirt2-SirReal interaction by means of a method that does not require a labeled ligand, we made use of the DNA nanolever-based switchSENSE ${ }^{\oplus}$ technology. ${ }^{[31]}$ This method is sensitive enough to monitor the binding of an unlabeled small molecule to a surface-tethered protein. ${ }^{[32]}$ In the experimental setup we used for our studies, Sirt2 was covalently bound to one strand of a double-stranded short DNA nanolever grafted to the surface of a gold microelectrode. The other strand is labeled with a fluorescent dye, in this case Cy3 (Figure $2 \mathrm{~A}$ ). In

B)
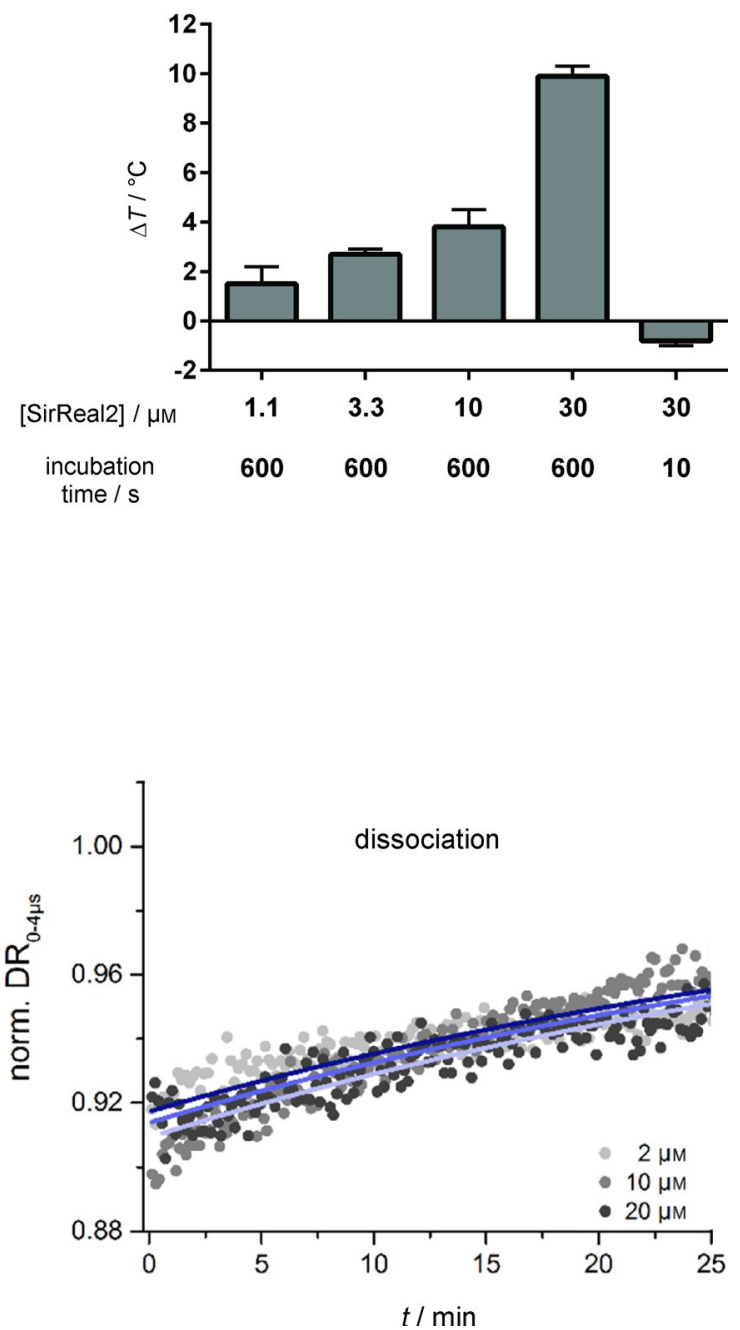

Figure 2. Probing the interaction between immobilized Sirt2 and unlabeled 2 by means of the switchSENSE technology. A) Cartoon representation of the experimental setup. B) Thermal stabilization of the immobilized Sirt2 evoked by different concentrations of $\mathbf{2}$. See Figure S3 for melting curves and their evaluation. Experimental details are provided in the Experimental Section. C) Association and dissociation curves of immobilized Sirt2 with 2 . Solid circles represent raw data, global fits are shown as lines. Experimental details are provided in the Experimental Section. 
general, such a setup can be used in two different measuring modes. In the static mode, ligand binding to the surface-tethered protein is monitored by changes in the fluorescence emission of the DNA-bound fluorophore as a direct consequence of the binding event. However, binding events that do not directly affect the fluorescence properties of the DNAbound fluorophore cannot be monitored in this mode. In the case of the SirReal-Sirt2 interaction, static-mode measurements did not yield any binding data that could be used for further evaluation (data not shown). Thus, we used the switchSENSE $^{\oplus}$ technology (dynamic mode) to further characterize the binding kinetics of the SirReal-Sirt2 interaction. The switchSENSE ${ }^{\ominus}$ technology makes use of alternating electric potentials that are applied to the gold microelectrode, which either attract or repel the negatively charged backbone of dsDNA nanolevers. This generates an oscillating change of orientation of the DNA nanolever, called switching. Due to a distancedependent, radiation-free energy transfer to the gold, the intensity of the fluorescent light emitted by the dye reports its distance from the gold surface. In other words, the closer the fluorophore is to the quenching gold surface, the less light is emitted. Processes that alter the hydrodynamic friction of the bound protein (e.g., ligand binding, conformational re-arrangement, thermal denaturation) affect the speed of DNA movement, which leads to a change in switching dynamics. The principle is depicted in detailed in Figure S2.

Prior to our kinetic measurements, we tested whether the immobilization procedure affected the structural integrity of Sirt2 and thus its ligand-binding properties. Therefore, we assessed the thermal stability of the tethered Sirt2 in the absence and presence of 2 by using switchSENSE ${ }^{\oplus}$ (Figure 2B). The thermal stabilization of the immobilized Sirt2 by $\mathbf{2}$, even at low-micromolar ligand concentrations, indicated proper folding and ligand binding properties. The thermal stabilization of tethered Sirt2 is concentration and time dependent (Figure $2 \mathrm{~B}$ ). Notably, when using the switchSENSE ${ }^{\oplus}$ technology, a significant thermal shift $\left(\Delta T \approx 3^{\circ} \mathrm{C}\right)$ could be detected at concentrations between 3.3 and $10 \mu \mathrm{M}$; a standard fluorescent thermal-shift assay (FTSA) with SYPRO Orange dye binding to untethered Sirt2 gave a similar shift $\left(\Delta T \approx 3^{\circ} \mathrm{C}\right)$ only at a higher compound concentration of $25 \mu \mathrm{M}$. $24 \mathrm{a}]$

According to the results of the thermal-stabilization assay, our kinetic measurements were performed at ligand concentrations between 2 and $20 \mu \mathrm{m}$. With switchSENSE ${ }^{\oplus}$ (dynamic mode), we observed a very slow dissociation of unlabeled 2 from immobilized Sirt2 (Figure 2C). Moreover, for the different concentrations of $2(2-20 \mu \mathrm{M})$, we obtained $k_{\text {on }}$ and $k_{\text {off }}$ rate constants as well as $K_{d}$ values (Table 1 ) in a highly similar range for this interaction compared to the previously reported data for the interaction between Sirt 2 and our labeled and immobilized SirReal-based affinity probe $\left(4, k_{\text {on }}=6.9 \pm 0.22 \times\right.$ $\left.10^{3} \mathrm{M}^{-1} \mathrm{~s}^{-1}, k_{\text {off }}=7.0 \pm 0.31 \times 10^{-4} \mathrm{~s}^{-1}, K_{\mathrm{d}}=0.10 \mu \mathrm{M}\right) .{ }^{[25]}$ By analyzing the recorded data set, we obtained rate constants of $k_{\text {on }}=$ $7.7 \pm 0.2 \times 10^{2} \mathrm{M}^{-1} \mathrm{~s}^{-1}, \quad k_{\text {off }}=4.1 \pm 0.1 \times 10^{-4} \mathrm{~s}^{-1}$; these give an overall dissociation constant of $K_{\mathrm{d}}=k_{\text {off }} / k_{\text {on }}=0.53 \pm 0.02 \mu \mathrm{M}$. This $K_{\mathrm{d}}$ value is strongly consistent with the reported $\mathrm{IC}_{50}$ value of $2 ; \mathrm{IC}_{50}=0.44 \mu \mathrm{m}^{[25]}$
Table 1 . The kinetic and affinity parameters $\left(k_{\mathrm{on}}, k_{\mathrm{off}}, K_{\mathrm{d}}\right)$ of the interaction between immobilized Sirt 2 and 2 determined by means of the switchSENSE ${ }^{\circ}$ technology.

\begin{tabular}{|lll|}
$k_{\text {off }}^{\text {[a] }}$ & $k_{\text {on }}^{\text {[a] }}$ & $K_{d}^{[b]}$ \\
\hline $4.1 \pm 0.1 \times 10^{-4} \mathrm{~s}^{-1}$ & $7.7 \pm 0.2 \times 10^{2} \mathrm{M}^{-1} \mathrm{~s}^{-1}$ & $0.53 \pm 0.02 \times 10^{-6} \mathrm{M}$ \\
\hline
\end{tabular}

[a] $k_{\text {on }}, k_{\text {off }}$ rate constants were determined by using a global single exponential fit, with the respective rate constant as global parameter. [b] $K_{d}$ was calculated from $k_{\text {off }} / k_{\text {on }}$ with err $K_{d}$ being the propagation of errors.

To further validate that the long residence time of the SirReals is an inherent characteristic of the ligand core itself and not caused by the additional $\mathrm{H}$-bond interaction of the triazole with Arg97, we determined the kinetic parameters for analogue 5 by means of the switchSENSE ${ }^{\circledast}$ technology (Figure S4). The obtained $k_{\text {off }}$ constant of $7.9 \pm 0.6 \times 10^{-4} \mathrm{~s}^{-1}$ is highly consistent with the $k_{\text {off }}$ constants determined for $4\left(k_{\text {off }}=7.0 \pm\right.$ $\left.0.31 \times 10^{-4} \mathrm{~s}^{-1}\right)$ and $2\left(k_{\text {off }}=4.1 \pm 0.1 \times 10^{-4} \mathrm{~s}^{-1}\right)$ determined by biolayer interferometry and the switchSENSE ${ }^{\oplus}$ technology, respectively. Thus, we were able to show that the previously reported long residence time of the SirReal-Sirt2 interaction is an inherent characteristic of the SirReal core itself and is neither a result of the incorporation of the triazole moiety nor an artefact of the applied measuring technology.

\section{Conclusion}

By proving that the SirReal core, which is sufficient to induce the structural rearrangement of Sirt2's active site, ${ }^{[24 a]}$ is the main driver of the slow off rate for this class of inhibitors, we have been able to add a further important piece of evidence that conformational adaption upon ligand binding greatly increases the residence time of the bound ligand, culminating in highly selective and high-affinity drug-target interactions. There is an emerging debate whether long residence time $\left(k_{\text {off }}\right)$ is really a better predictor of success in drug development than mere potency or affinity $\left(I C_{50}\right.$ or $\left.K_{d}\right){ }_{i}^{[33]}$ our study suggests that a long residence time as a consequence of an induced-fit mechanism can be an important driver of target selectivity, which is one of the most crucial parameters in modern drug discovery.

\section{Experimental Section}

Chemicals: SirReal analogues $\mathbf{2}$ and $\mathbf{5}$ were synthesized according to published procedures. ${ }^{[24,25]}$ The purity of the compounds was confirmed by HPLC analysis to be at least $95 \%$.

Protein production and purification: Human Sirt $2_{25-389}$ was expressed with an N-terminal hexahistidine tag, ${ }^{[34]}$ and purified with minor modifications according to Neugebauer et al. ${ }^{[35]}$

SwitchSENSE ${ }^{\oplus}$ Sirt2-SirReal2 thermal stabilization experiments: The thermal stabilization of immobilized Sirt2 evoked by different concentrations (1.1-30 $\mu \mathrm{M})$ of 2 was determined by using switchSENSE $^{\oplus}$ technology on a DRX instrument (Dynamic Biosensors $\mathrm{GmbH}$, Martinsried, Germany). ${ }^{[36]}$ In this experimental assay setup, Sirt2 was immobilized on the switchSENSE ${ }^{\circledast}$ chip (MPC-48-2-Y1-S) 
biosensor surface, while $\mathbf{2}$ was injected as the analyte in solution for the indicated duration. For immobilization on the biosensor surface, Sirt2 was covalently coupled to single-stranded 48-mer DNA complementary in sequence to the ssDNA functionalized on the biosensor surface by using amine chemistry (amine coupling kit CK-NH2-1-B48). The Sirt2-DNA conjugate was hybridized to the covalently immobilized single-stranded surface DNA. All experiments were carried out in TE20-5 buffer $(10 \mathrm{~mm}$ Tris $\cdot \mathrm{HCl}, \mathrm{pH} 7.4$, $20 \mathrm{~mm} \mathrm{NaCl}, 5 \mathrm{~mm} \mathrm{MgCl}, 50 \mu \mathrm{m}$ EGTA, $50 \mu \mathrm{m}$ EDTA, 0.05\% Tween 20) and monitored in dynamic measurement mode. After Sirt2 had been immobilized, $\mathbf{2}$ was injected. Melting curves were detected during a ramp from 25 to $75^{\circ} \mathrm{C}\left(5^{\circ} \mathrm{C} \mathrm{min}{ }^{-1}\right)$. A melting curve for blunt DNA was recorded and subtracted. Melting temperatures were determined by using a Boltzmann Fit, Origin 2015 software (OriginLab Corporation, Northampton, MA, USA).

SwitchSENSE ${ }^{\oplus}$ Sirt2-SirReal kinetic experiments: The kinetic and affinity parameters $\left(k_{\mathrm{on}}, k_{\mathrm{off}}, K_{\mathrm{d}}\right)$ of the interaction between Sirt2 and the SirReal analogues 2 and $\mathbf{5}$ were determined at concentrations of 2,10 , and $20 \mu \mathrm{M}$ by using switchSENSE ${ }^{\oplus}$ technology (dynamic mode) on a DRX instrument (Dynamic Biosensors). ${ }^{[36]}$ In this experimental assay setup, Sirt2 was immobilized on the switchSENSE ${ }^{\oplus}$ chip (MPC-48-2-Y1-S) biosensor surface, while the SirReal analogue was injected as the analyte in solution. For immobilization on the biosensor surface, Sirt2 was covalently coupled to 48mer ssDNA complementary in sequence to the ssDNA functionalized on the biosensor surface by using amine chemistry (amine coupling kit CK-NH2-1-B48). The Sirt2-DNA conjugate was hybridized to the covalently immobilized single-stranded surface DNA. All experiments were carried out in PE40 buffer $\left(10 \mathrm{mM} \mathrm{Na}_{2} \mathrm{HPO}_{4} /\right.$ $\mathrm{NaH}_{2} \mathrm{PO}_{4}, \mathrm{pH} 7.4,40 \mathrm{~mm} \mathrm{NaCl}, 50 \mu \mathrm{m}$ EGTA, $50 \mu \mathrm{M}$ EDTA, $0.05 \%$ Tween 20) and monitored in dynamic measurement mode. After immobilization of Sirt2, the SirReal analogue was injected. The obtained data were fitted and evaluated by using a global single exponential fit, Origin 2015 software (OriginLab Corporation, Northampton, MA, USA).

Definition of "dynamic response" (DR): During kinetic measurements as well as melting experiments, alternating potentials are applied to induce a switching motion of the DNA nanolevers (dynamic measurement mode). The switching motion is resolved by time-correlated single photon counting. ${ }^{[36]}$ Beside the resulting fluorescence transition curves of upward and downward motion of DNA nanolevers (describing the phase of attraction of levers towards a positively charged gold electrode and repulsion from a negatively charged gold electrode), it is more practical to define a dynamic response parameter (DR). ${ }^{[36]}$ The general calculation of dynamic response units (DRU) is shown in Equation (1). Small molecule kinetic measurements use an integrated fluorescence signal of the first $4 \mu$ s of the upward motion of nanolevers, indicated as " $D R_{0-4 \mu s}$ ". Melting of proteins is determined with a dynamic response calculated from the first $10 \mu \mathrm{s}\left(\right.$ (" $\mathrm{DR}_{0-10 \mu \mathrm{s}}$ ").

$\mathrm{DR}_{t 1 \text { up }}^{t 2}=\int_{t 1}^{t 2} F_{\text {norm }} \mathrm{d} t, \mathrm{DR}_{t 1 \text { down }}^{t 2}=\int_{t 1}^{t 2}\left(1-F_{\text {norm }}\right) \mathrm{d} t$

\section{Acknowledgements}

M.S. (SCHI 1408/2-1) and M.J. (Ju295/14-1) are supported by the Deutsche Forschungsgemeinschaft (DFG).

\section{Conflict of Interest}

H.D. is an application scientist working for Dynamic Biosensors $\mathrm{GmbH}$.

Keywords: deacylases - drug design - epigenetics - protein modifications $\cdot$ sirtuins

[1] C. T. Walsh, S. Garneau-Tsodikova, G. J. Gatto, Angew. Chem. Int. Ed. 2005, 44, 7342-7372; Angew. Chem. 2005, 117, 7508-7539.

[2] T. Kouzarides, EMBO J. 2000, 19, 1176-1179.

[3] E. Verdin, M. Ott, Nat. Rev. Mol. Cell Biol. 2015, 16, 258-264.

[4] C. Choudhary, B. T. Weinert, Y. Nishida, E. Verdin, M. Mann, Nat. Rev. Mol. Cell Biol. 2014, 15, 536-550.

[5] J. Baeza, M. J. Smallegan, J. M. Denu, ACS Chem. Biol. 2015, 10, 122 128.

[6] A. J. M. De Ruijter, A. H. Van Gennip, H. N. Caron, S. Kemp, A. B. P. Van Kuilenburg, Biochem. J. 2003, 370, 737-749.

[7] B. D. Sanders, B. Jackson, R. Marmorstein, Biochim. Biophys. Acta Proteins Proteomics 2010, 1804, 1604-1616.

[8] B. J. North, B. L. Marshall, M. T. Borra, J. M. Denu, E. Verdin, Mol. Cell 2003, 11, $437-444$.

[9] B. J. North, M. A. Rosenberg, K. B. Jeganathan, A. V. Hafner, S. Michan, J. Dai, D. J. Baker, Y. N. Cen, L. E. Wu, A. A. Sauve, J. M. van Deursen, A. Rosenzweig, D. A. Sinclair, EMBO J. 2014, 33, 1438-1453.

[10] H. Vaziri, S. K. Dessain, E. N. Eagon, S. I. Imai, R. A. Frye, T. K. Pandita, L. Guarente, R. A. Weinberg, Cell 2001, 107, 149-159.

[11] a) M. Ishfaq, K. Maeta, S. Maeda, T. Natsume, A. Ito, M. Yoshida, Biosci. Biotechnol. Biochem. 2012, 76, 2165-2167; b) A. A. Shah, A. Ito, A. Nakata, M. Yoshida, Bio.l Pharm. Bull. 2016, 39, 1739-1742.

[12] K. M. Rothgiesser, S. Erener, S. Waibel, B. Luscher, M. O. Hottiger, J. Cell Sci. 2010, 123, $4251-4258$.

[13] R. M. de Oliveira, J. Sarkander, A. G. Kazantsev, T. F. Outeiro, Front. Pharmacol. 2012, 3, 82.

[14] B. Beirowski, J. Gustin, S. M. Armour, H. Yamamoto, A. Viader, B. J. North, S. Michan, R. H. Baloh, J. P. Golden, R. E. Schmidt, D. A. Sinclair, J. Auwerx, J. Milbrandt, Proc. Natl. Acad. Sci. USA 2011, 108, E952-E961.

[15] a) T. F. Pais, E. M. Szego, O. Marques, L. Miller-Fleming, P. Antas, P. Guerreiro, R. M. de Oliveira, B. Kasapoglu, T. F. Outeiro, EMBO J. 2013, 32, 2603-2616; b) H. A. Eskandarian, F. Impens, M. A. Nahori, G. Soubigou, J. Y. Coppee, P. Cossart, M. A. Hamon, Science 2013, 341, 1238858.

[16] Y. B. Teng, H. Jing, P. Aramsangtienchai, B. He, S. Khan, J. Hu, H. N. Lin, Q. Hao, Sci. Rep. 2015, 5, 8529.

[17] A. Szabo, J. Olah, S. Szunyogh, A. Lehotzky, T. Szenasi, M. Csaplar, M. Schiedel, P. Low, M. Jung, J. Ovadi, Sci. Rep. 2017, 7, 17070.

[18] S. H. Park, Y. M. Zhu, O. Ozden, H. S. Kim, H. Y. Jiang, C. X. Deng, D. Gius, A. Vassilopoulos, Transl. Cancer Res. 2012, 1, 15-21.

[19] G. Donmez, T. F. Outeiro, EMBO Mol. Med. 2013, 5, 344-352.

[20] H. S. Kim, A. Vassilopoulos, R. H. Wang, T. Lahusen, Z. Xiao, X. L. Xu, C. L. Li, T. D. Veenstra, B. Li, H. T. Yu, J. F. Ji, X. W. Wang, S. H. Park, Y. I. Cha, D. Gius, C. X. Deng, Cancer Cell 2011, 20, 487-499.

[21] a) V. Chopra, L. Quinti, J. Kim, L. Vollor, K. L. Narayanan, C. Edgerly, P. M. Cipicchio, M. A. Lauver, S. H. Choi, R. B. Silverman, R. J. Ferrante, S. Hersch, A. G. Kazantsev, Cell Rep. 2012, 2, 1492-1497; b) A. Bobrowska, G. Donmez, A. Weiss, L. Guarente, G. Bates, PLoS One 2012, 7, e34805.

[22] a) L. Serrano, P. Martinez-Redondo, A. Marazuela-Duque, B. N. Vazquez, S. J. Dooley, P. Voigt, D. B. Beck, N. Kane-Goldsmith, Q. Tong, R. M. Rabanal, D. Fondevila, P. Munoz, M. Kruger, J. A. Tischfield, A. Vaquero, Genes Dev. 2013, 27, 639-653; b) P. Y. Liu, N. Xu, A. Malyukova, C. J. Scarlett, Y. T. Sun, X. D. Zhang, D. Ling, S. P. Su, C. Nelson, D. K. Chang, J. Koach, A. E. Tee, M. Haber, M. D. Norris, C. Toon, I. Rooman, C. Xue, B. B. Cheung, S. Kumar, G. M. Marshall, A. V. Biankin, T. Liu, Cell Death Differ. 2013, 20, 503-514.

[23] M. Schiedel, D. Robaa, T. Rumpf, W. Sippl, M. Jung, Med. Res. Rev. 2018, 38, 147-200.

[24] a) T. Rumpf, M. Schiedel, B. Karaman, C. Roessler, B. J. North, A. Lehotzky, J. Olah, K. I. Ladwein, K. Schmidtkunz, M. Gajer, M. Pannek, C. Steegborn, D. A. Sinclair, S. Gerhardt, J. Ovadi, M. Schutkowski, W. Sippl, O. 
Einsle, M. Jung, Nat. Commun. 2015, 6, 6263; b) M. Schiedel, T. Rumpf, B. Karaman, A. Lehotzky, J. Olah, S. Gerhardt, J. Ovadi, W. Sippl, O. Einsle, M. Jung, J. Med. Chem. 2016, 59, 1599-1612.

[25] M. Schiedel, T. Rumpf, B. Karaman, A. Lehotzky, S. Gerhardt, J. Ovadi, W. Sippl, O. Einsle, M. Jung, Angew. Chem. Int. Ed. 2016, 55, 2252-2256; Angew. Chem. 2016, 128, 2293-2297.

[26] J. L. Feldman, K. E. Dittenhafer-Reed, N. Kudo, J. N. Thelen, A. Ito, M. Yoshida, J. M. Denu, Biochemistry 2015, 54, 3037-3050.

[27] a) S. Sundriyal, S. Moniot, Z. Mahmud, S. Yao, P. Di Fruscia, C. R. Reynolds, D. T. Dexter, M. J. E. Sternberg, E. W. F. Lam, C. Steegborn, M. J. Fuchter, J. Med. Chem. 2017, 60, 1928-1945; b) P. Mellini, Y. Itoh, H. Tsumoto, Y. Li, M. Suzuki, N. Tokuda, T. Kakizawa, Y. Miura, J. Takeuchi, M. Lahtela-Kakkonene, T. Suzuki, Chem. Sci. 2017, 8, 6400-6408; c) D Robaa, D. Monaldi, N. Wossner, N. Kudo, T. Rumpf, M. Schiedel, M. Yoshida, M. Jung, Chem. Rec. 2018, 18, $1701-1707$.

[28] A. Basavapathruni, L. Jin, S. R. Daigle, C. R. Majer, C. A. Therkelsen, T. J. Wigle, K. W. Kuntz, R. Chesworth, R. M. Pollock, M. P. Scott, M. P. Moyer, V. M. Richon, R. A. Copeland, E. J. Olhava, Chem. Biol. Drug Des. 2012, $80,971-980$.

[29] R. A. Copeland, Future Med. Chem. 2011, 3, 1491-1501.

[30] a) R. A. Copeland, D. L. Pompliano, T. D. Meek, Nat. Rev. Drug Discovery 2006, 5, 730-739; b) R. A. Copeland, Nat. Rev. Drug Discovery 2016, 15, $87-95$.
[31] J. Knezevic, A. Langer, P. A. Hampel, W. Kaiser, R. Strasser, U. Rant, J. Am. Chem. Soc. 2012, 134, 15225-15228.

[32] E. Kyriakou, S. Schmidt, G. T. Dodd, K. Pfuhlmann, S. E. Simonds, D. Lenhart, A. Geerlof, S. C. Schriever, M. De Angelis, K. W. Schramm, O. Plettenburg, M. A. Cowley, T. Tiganis, M. H. Tschop, P. T. Pfluger, M. Sattler, A. C. Messias, J. Med. Chem. 2018, 61, 11144-11157.

[33] R. H. A. Folmer, Drug Discovery Today 2018, 23, 12-16.

[34] B. J. North, B. Schwer, N. Ahuja, B. Marshall, E. Verdin, Methods 2005, 36, $338-345$.

[35] R. C. Neugebauer, U. Uchiechowska, R. Meier, H. Hruby, V. Valkov, E. Verdin, W. Sippl, M. Jung, J. Med. Chem. 2008, 51, 1203-1213.

[36] A. Langer, P. A. Hampel, W. Kaiser, J. Knezevic, T. Welte, V. Villa, M. Maruyama, M. Svejda, S. Jahner, F. Fischer, R. Strasser, U. Rant, Nat. Commun. 2013, 4, 2099.

Manuscript received: August 23, 2019

Revised manuscript received: October 31, 2019

Accepted manuscript online: November 6, 2019

Version of record online: January 22, 2020 